\title{
Feasibility and efficacy of text messaging to promote care among trauma patients screened for HIV at an urban emergency department in Tanzania
}

Gimbo M. Hyuha', Hendry R. Sawe ${ }^{1,2^{*}}$ D, Said Kilindimo ${ }^{1,2}$, Raya Y. Mussa ${ }^{1}$, Masuma A. Gulamhussein ${ }^{1}$, Shamila S. Rwegoshora', Frida Shayo ${ }^{1}$, Winnie Mdundo', Juma A. Mfinanga ${ }^{1,2}$ and Ellen J. Weber ${ }^{1,3}$

\begin{abstract}
Background: Due to the high prevalence of human immunodeficiency virus (HIV) in Tanzania, provider-initiated HIV testing for patients attending any health care setting is recommended. However, follow-up and linkage to care by those tested remain poor. We determined the feasibility and efficacy of text messaging to promote follow-up among otherwise healthy trauma patients who underwent provider-initiated HIV testing and counseling at an emergency department (ED) in Tanzania.

Material and methods: This randomized controlled trial (RCT) was conducted at Muhimbili National Hospital (MNH) ED between September 2019 and February 2020. Adult trauma patients consenting to HIV testing and follow-up text messaging were randomized to standard care (pre-test and post-test counseling) or standard care plus a series of three short message service (SMS) text message reminders for follow-up in an HIV clinic, if positive, or for retesting, if negative. Investigators blinded to the study assignment called participants 2 months after the ED visit if HIV-positive or 4 months if HIV-negative. We compared the proportion of people in the intervention and control groups completing recommended follow-up. Secondary outcomes were the proportion of patients agreeing to testing, proportion of patients agreeing to receiving text messages, and the proportion of HIV-positive and HIV-negative patients in each study arm who followed up.
\end{abstract}

\footnotetext{
* Correspondence: hendry_sawe@yahoo.com

'Emergency Medicine Department, Muhimbili University of Health and Allied Sciences, P.O. Box 65001, Dar es Salaam, Tanzania

2Emergency Medicine Department, Muhimbili National Hospital, Dar es Salaam, Tanzania

Full list of author information is available at the end of the article
}

C C The Author(s). 2021 Open Access This article is licensed under a Creative Commons Attribution 4.0 International License, which permits use, sharing, adaptation, distribution and reproduction in any medium or format, as long as you give appropriate credit to the original author(s) and the source, provide a link to the Creative Commons licence, and indicate if changes were made. The images or other third party material in this article are included in the article's Creative Commons licence, unless indicated otherwise in a credit line to the material. If material is not included in the article's Creative Commons licence and your intended use is not permitted by statutory regulation or exceeds the permitted use, you will need to obtain permission directly from the copyright holder. To view a copy of this licence, visit http://creativecommons.org/licenses/by/4.0/ The Creative Commons Public Domain Dedication waiver (http://creativecommons.org/publicdomain/zero/1.0/) applies to the data made available in this article, unless otherwise stated in a credit line to the data. 
Results: Of the 290 patients approached, 255 (87.9\%) opted-in for testing and agreed to receive a text message. The median age of the study population was 29 [IQR 24-40] years. There were 127 patients randomized to the intervention group and 128 to the control group. The automated SMS system verified that 381 text messages in total were successfully sent. We traced 242 (94.9\%) participants: 124 (51.2\%) in the intervention group and 18 (488\%) in the control group. A total of 100 (39.2\%) subjects reported completing a follow-up visit, of which 77 (60.6\%) were from the intervention group and 23 (17.9\%) were from the control group ( $R R=3.4,95 \% \mathrm{Cl} 2.3-5.0$ ). This resulted in a number needed to treat (NNT) of 2.3. Of the 246 HIV-negative participants, $37 \%$ underwent repeat screening: $59 \%$ of those in the intervention group and $16 \%$ in the control group (RR $=3.7, P=\langle 0.0001$, NNT 2.3). Among the nine positive patients, all five in the intervention group and only three in the controls had follow-up visits.

Conclusion: Automated text message is a feasible and effective way to increase follow-up in HIV-tested individuals in a limited income country.

Keywords: HIV, AIDS, SMS, Text message, Reminder, Cell phone, Opt-out, PITC, Trauma, Emergency department, Tanzania

\section{Background}

HIV continues to be a major global public health problem, claiming more than 35 million lives thus far. In 2017, 940,000 people died from HIV-related causes globally [1]. Sub-Saharan Africa, including Tanzania, is the most affected region in the world, with 25.8 million people living with HIV in 2017. Two-thirds of the global total of new HIV infections occur in sub-Saharan Africa [2]. In 2016, 1.4 million people were living with HIV in Tanzania. This equates to an estimated HIV prevalence of $4.7 \%$ [3].

Routine provider-initiated testing, with the opportunity for patients to opt-out, has been shown to identify people living with HIV/AIDS earlier, enroll them into care, and reduce mortality [4-6]. In Tanzania, the National Comprehensive Guideline for HIV Testing and Counseling of 2017 recommends routine testing and screening for HIV/AIDS in all health centers [6]. However, follow-up is poor in these patients after testing [5, 7, 8]. Failure to attend medical appointments among persons living with HIV has been associated with poor health outcomes $[9,10]$.

Text messaging via SMS is a potential intervention for improving retention and compliance with recommended care, by providing medication and appointment reminders [7, 8, 11-17]. Studies on the feasibility and efficacy of using text messaging via SMS in promoting care have been performed in many countries; almost all have shown an improvement in health care behaviors and disease outcomes $[7,8,11-17]$. Nevertheless, there are only a few studies on the feasibility and efficacy of using SMS as a means to promote care in sub-Saharan Africa and none in Tanzania $[7,8,15]$. Further, unlike high-income countries (HICs) where individuals frequently encounter the health care system due to established primary care systems, in low- and middle-income countries (LMICs), many people with HIV do not know they have it and will not be tested. The ability of text messaging to improve compliance among otherwise asymptomatic patients or those that need repeat screening has not been studied in any country.

The Muhimbili National Hospital - Emergency Department (MNH-ED) created a protocol for rapid HIV testing of trauma patients in 2018. Trauma patients are usually healthy and would not otherwise seek health care where provider-initiated HIV testing might occur. A pilot study in our ED found that these patients were willing to be tested, but of those who were HIV-positive, only half sought follow-up care [6]. We therefore tested the feasibility and efficacy of using automated text messaging to promote follow-up care among otherwise healthy patients screened for HIV at the MNH-ED.

\section{Methodology}

\section{Study design}

This was a single-center, randomized, blinded controlled trial of adult trauma patients presenting to the Emergency Department of Muhimbili National Hospital (MNH-ED) between September 2019 and February 2020 who agreed to provider-initiated, rapid HIV testing.

\section{Study setting}

Muhimbili National Hospital (MNH) is a tertiary referral hospital with a level 1 trauma center located in Dar es Salaam, Tanzania. The hospital has a bed capacity of 1500, averaging 1000 to 1200 admissions per week. The MNH-ED is the first public emergency department (ED) in the country and opened in 2010 [18]. It is staffed with emergency physicians, postgraduate students in an emergency medicine training program, medical officers, critical care nurses, and nursing officers. The MNH-ED is the entry point to the hospital for most of the patients 
attending $\mathrm{MNH}$ and sees an average of 200 patients daily, with an admission rate of $65 \%$. Acutely ill patients are received at the ED, resuscitated, and stabilized before being transferred to the appropriate ward.

\section{Participants}

All adult patients with trauma attending the MNH-ED were eligible for the study. To be included, patients had to agree to be tested for HIV and be willing and able to receive text messages on their mobile phones. Patients were excluded if they were below 18 years old, had a Glasgow Coma Scale score below 15, known to be HIVpositive with evidence of either a treatment card or medications, were clinically unstable, did not own a cell phone or phone was not capable of receiving an SMS, or had been previously enrolled in the study.

\section{Study protocol}

The study was registered in the Pan African Clinical Trials Registry (PACTR), with the trial registration number PACTR201909858930669. Researchers actively walked through the ED to identify trauma patients and used the tracking board of the ED's electronic medical record $\left(W^{2}\right.$ llsoft $\left.{ }^{\mathrm{m}}\right)$ : to identify patients triaged with a complaint related to trauma. Researchers screened patients for eligibility and willingness to have an HIV test and receive text messages. They then consented the patients to the study. Research assistants captured baseline patient data using a structured case report form; data were then transferred to an online data storage platform (Redcap ${ }^{\mathrm{rm}}$ ). A trained nurse provided pre-test and post-test counseling to all patients tested for HIV. Standard trauma treatment for injuries was performed at the discretion of the treating physician.

\section{Randomization and blinding}

Prior to beginning enrollment, we created a computergenerated randomization scheme that ensured equal numbers of participants in the intervention and control groups [19]. Immediately after consent, patients were randomly assigned to the intervention or control group. Allocation was concealed from patients and research assistants; research assistants knew that the patient was assigned to group A or B but did not know which was the control or intervention group.

\section{Intervention}

We used an automated Short Message Service (SMS) system to send texts at scheduled times to the patients in the intervention arm. Patient information and their group assignment were transferred to the Extrateq ${ }^{\text {tw }}$-automated SMS dispatch program. SMS messages were sent only to patients that had been randomized to the intervention arm of the study. The program was set to deliver text messages three times for everyone in the intervention group, once per week for HIV-positive patients, and once per month for HIV-negative patients, starting from the day of the ED visit. SMS content was sent in Swahili with an English translation reading: "Dear EMD client, this is a reminder that it is important for your health that you visit the nearest hospital to you., Thank you in advance and have a good day." To assess the fidelity of the intervention, automated SMS system was queried at the end of the study to determine how many texts were successfully sent.

\section{Patient follow-up}

Follow-up phone calls were made by research assistants or the primary investigator 2 months after the ED visit if the subject was HIV-positive or months after the visit if the patient was HIV-negative. The researchers making the calls were blinded to the arm of the study the patient. All traced participants were asked during the follow-up phone call whether they received a message at least once and to report whether they attended their follow-up Care and Treatment Centre (CTC) clinic (if HIV-positive) or went for repeat HIV screening (if HIVnegative). Those who were not traced were considered lost to follow-up. Follow-up interview data was entered into the patient's initial questionnaire and subsequently uploaded to the data storage platform.

\section{Outcomes}

The primary outcome was the proportion of patients who reported attending follow-up visits either to the CTC clinic (HIV-positive) or another site for repeat HIV testing (HIV-negative). Based on a previous study by Mugo et al. [8] where compliance in the intervention and control groups was $59 \%$ and $41 \%$, respectively, we determined the minimum sample size required was 240 (120 in each group). Secondary outcomes were the proportion of patients agreeing to testing, the proportion of patients agreeing to receive text messages, and the proportion of HIV-positive and HIV-negative patients in each study arm who followed up.

\section{Data analysis}

Data was coded and imported into Research Electronic Data Capture (REDCap). Statistical Package for Social Science (IBM SPSS version 25, IBM, LTD, Carolina, USA) was used for analysis. Relevant frequencies and tables were generated for all variables. The means, proportions, medians, and interquartile range were calculated for continuous variables according to their distribution. An intent-to-treat analysis was used to arrive at our results.

The primary outcome was analyzed using Pearson's chi-square test and the difference shown using relative 
risks (RR) with 95\% confidence intervals (CI) and number needed to treat (NNT). The secondary outcomes were compared using Pearson's chi-square test or Fisher's exact test, and proportions or means were used to report secondary outcomes and differences shown by RR. Statistical analysis was two-tailed, and a $P$ value of less than 0.05 was considered statistically significant.

\section{Results}

\section{Recruitment and baseline characteristics of the study} population

During the study period, 1804 adult trauma patients were seen in the MNH-ED. Of these patients, $70.7 \%$ were excluded due to severe injuries with instability (Fig. 1). The remaining were consecutively screened ( $N=529)$, and 290 met the eligibility criteria. Of these, 255 (87.9\%) consented to taking an HIV test and participating in the study. The reason for not participating was most commonly stress from the traumatic experience of being involved in an accident and not being emotionally ready to take on more stress if the results of the HIV test were positive. Of the 255 enrolled patients, we achieved phone followup for 242 (94.8\%) patients.

Most participants were male (83\%), and most participants were young adults with a median age of 29 years with an IQR of 24--40 years (Table 1). Approximately half were married; the majority $(82.4 \%)$ had achieved primary education as their highest educational level. Most (82.4\%) of the study population was referred from outside hospitals, and $85 \%$ of the study population had no health care insurance. The majority of participants were employed by others (i.e., hired staff, 60.4\%). Of the 255 enrolled, 9 (6\%) tested HIV-positive.

There were 128 (50.2\%) participants in the control group and 127 (49.8\%) participants in the intervention group (Fig. 1). There were more males in the intervention group and more females in the control group. More of the elderly participants were randomized into the intervention arm (Table 1). There were 5 HIV-positive and 122-HIV negative patients in the intervention group and 4 HIV-positive and $124 \mathrm{HIV}$-negative patients in the control group.

A review of the report from Extrateq ${ }^{\mathrm{TM}}$ showed that 381 text messages were successfully sent, consistent with 3 calls per person in the intervention group.

\section{Compliance with follow-up for recommended care}

A total of 242 (94.9\%) participants were reached by phone in follow-up. Among the 13 (5\%) that were lost to follow-up, three were in the intervention group and ten were in the control group. All interventional group participants that were reached reported that they had received the text message at least once and all individuals in the control group denied having received any messages.

Among the 242 reached, 100 (41.3\%) participants reported they completed follow-up care according to their respective HIV status; $77 \%$ of those who completed care were in the intervention group and $23 \%$ were in the control group $(P=<0.0001)$. Relative risk (RR) for attending follow-up care was 3.4 (CI 2.3-5.0) if the patient had received a text. The number of patients needed to treat (NNT) (i.e., receive a text) to obtain one follow-up visit was 2.3 (95\% CI 1.9-3.1) (Table 2).

\section{Follow-up by HIV status}

In the HIV-negative population, $37.4 \%$ of patients underwent repeat screening in the recommended time period. Repeat screening was significantly more frequent in the intervention group compared with the control group (59.0\% vs $16.1 \%$, respectively, $P<0.0001$ ). Sending a text message increased the likelihood of follow-up in HIVnegative patients with a $\mathrm{RR}=3.65$ compared to no text, and NNT was 2.3 (95\% CI 1.9-3.1) (Table 2).

All 9 HIV-positive patients were reached by the study investigators. Among these, 8 (88.9\%) reported having attended an initial CTC visit. All 5 in the intervention group and 3 out of 4 in the control group had attended an initial CTC visit. The numbers were too small for statistical analysis (Table 2).

\section{Discussion}

This RTC tested the feasibility and efficacy of text messaging to promote follow-up care among otherwise healthy trauma patients screened for HIV seen at an emergency department in a limited income country. We found that most patients approached were willing to be tested and receive SMS messages. Follow-up with recommended care was significantly greater in those who received the text messages compared to those who did not, $\mathrm{RR}=3.4$.

Previous studies evaluating the effect of SMS in the promotion of patient care to improve compliance with care in HIV and other chronic illnesses [7, 8, 11-17]. A 2017 systematic review by Daher et al. including 99 studies in Africa, Asia, Europe, and America assessed whether digital innovations were feasible, acceptable, and had a general impact on the promotion of care in HIV and other sexually transmitted diseases. The review concluded that these innovations were feasible, acceptable, and had an impact on the promotion of care [13]. A 2017 meta-analysis by Fontelo et al., which analyzed 34 different studies globally, showed that text message was a valuable tool to increase general HIV-related compliance for clinic attendance and medication adherence [14]. 


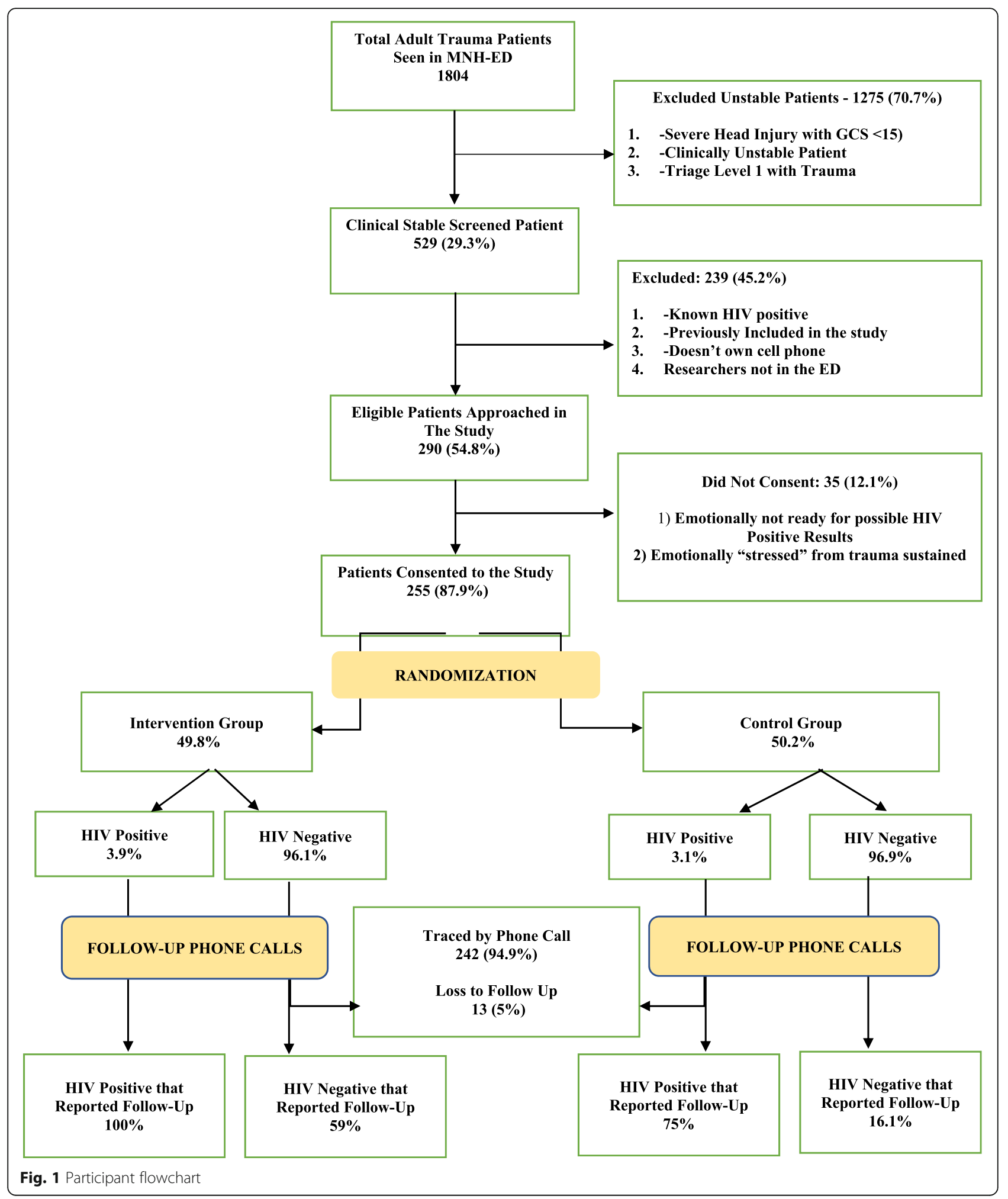

Our study had several unique aspects. While prior RCTs have been conducted using digital interventions like text messages to improve the care of HIV patients in low- and middle-income countries (LMICs) [7, 8, 10,
15], most of these studies were confined to patients known to have or suspected to have HIV due to symptoms. While our study assessed the value of this intervention in asymptomatic patients who had not sought 
Table 1 Demographic characteristics of the study population

\begin{tabular}{|c|c|c|c|}
\hline Demographic characteristic & Total, $N=255$ & Control group, $n(\%), N=128$ & Intervention group, $n(\%), N=127$ \\
\hline \multicolumn{4}{|l|}{ Sex } \\
\hline Male & 211 & $103(48.8 \%)$ & $108(51.2 \%)$ \\
\hline Female & 44 & $25(56.8 \%)$ & $19(43.2 \%)$ \\
\hline \multicolumn{4}{|l|}{ Age groups } \\
\hline $18-35$ years & 175 & $92(52.6 \%)$ & $83(47.4 \%)$ \\
\hline $36-55$ years & 64 & $32(50.0 \%)$ & $32(50.0 \%)$ \\
\hline$>55$ years & 16 & $4(25.0 \%)$ & $12(75.0 \%)$ \\
\hline \multicolumn{4}{|l|}{ Marital status } \\
\hline Divorced & 11 & $5(45.5 \%)$ & $6(54.5 \%)$ \\
\hline Married & 144 & $73(50.7 \%)$ & $71(49.3 \%)$ \\
\hline Single & 97 & 49 (50.5\%) & $48(49.5 \%)$ \\
\hline Widowed & 3 & $1(33.3 \%)$ & $2(66.7 \%)$ \\
\hline \multicolumn{4}{|l|}{ Level of education } \\
\hline College & 27 & $12(44.4 \%)$ & $15(55.6 \%)$ \\
\hline Informal & 10 & $4(40.0 \%)$ & $6(60.0 \%)$ \\
\hline Primary & 139 & $69(49.6 \%)$ & $70(50.4 \%)$ \\
\hline Secondary & 74 & $39(52.7 \%)$ & $35(47.3 \%)$ \\
\hline University & 5 & $4(80.0 \%)$ & 1 (20.0\%) \\
\hline \multicolumn{4}{|l|}{ Occupation } \\
\hline Employed & 154 & 72 (46.8\%) & $82(53.2 \%)$ \\
\hline Student & 5 & $1(20.0 \%)$ & $4(80.0 \%)$ \\
\hline Unemployed & 89 & $52(58.4 \%)$ & $37(41.6 \%)$ \\
\hline Unskilled Labor & 7 & $3(42.9 \%)$ & $4(57.1 \%)$ \\
\hline \multicolumn{4}{|l|}{ Insurance status } \\
\hline Insured & 38 & $16(42.1 \%)$ & 22 (57.9\%) \\
\hline Non-insured & 217 & $112(51.6 \%)$ & 105 (48.4\%) \\
\hline \multicolumn{4}{|l|}{ Referral status } \\
\hline Referral & 210 & $105(50.0 \%)$ & $105(50.0 \%)$ \\
\hline Non-referral & 45 & $23(51.1 \%)$ & 22 (48.9\%) \\
\hline \multicolumn{4}{|l|}{ HIV status } \\
\hline Negative & 246 & $124(50.4 \%)$ & $122(49.6 \%)$ \\
\hline Positive & 9 & $4(44.4 \%)$ & $5(55.6 \%)$ \\
\hline
\end{tabular}

out HIV screening. This study also included HIVnegative patients and found that SMS significantly improved compliance with repeat testing at three months. A Kenyan study by Mugo et al. [8] comparing the impact of text messages, phone calls, and in-person appointment reminders on the rate of repeat screening of HIV also found that text messages increased the likelihood of accessing follow-up services. However, that study included only patients who had presented to a clinic because of their medical symptoms.

Another unique aspect of our study was the use of pre-programmed, automated SMS which allows messages to be sent to specific individuals at specified times.
The intervention does not require having someone to remember to send the messages. The intervention had high fidelity: 381 messages were logged as sent, participants in the intervention arm remembered receiving a message, and no patients in the control arm reported receiving the SMS.

This study also shows the importance of providerinitiated counseling and testing for every patient entering the hospital. Among patients without medical complaints, and who would have not presented to a medical facility other than for trauma, we found a HIV incidence of $3.5 \%$. This is substantially higher than in many EDbased screening programs in high-income countries. 
Table 2 Follow-up visits according to treatment arm

\begin{tabular}{|c|c|c|c|}
\hline & Number & Total follow up services in all study patients, $N(\%)$ & $P$ value \\
\hline Intervention group & 127 & $77(60.6 \%)$ & $<0.0001$ \\
\hline Control group & 128 & 23(17.9\%) & \\
\hline \multicolumn{4}{|c|}{ *RR 3.4 (Cl 2.3-5.0), NNT 2.3 (95\% Cl 3.1-1.9) } \\
\hline & N & Repeated HIV screening in HIV negatives, $N(\%)$ & $P$ value \\
\hline Intervention group & 122 & $72(59.0 \%)$ & $<0.0001$ \\
\hline Control group & 124 & $20(16.1 \%)$ & \\
\hline \multicolumn{4}{|c|}{ *RR 3.7 (Cl 2.4-5.6), NNT 2.3 (95\% Cl 3.1-1.9) } \\
\hline & N & Follow up visit to CTC in HIV positives, $N(\%)$ & Fisher exact probability \\
\hline Intervention group & 5 & $5(100 \%)$ & N/A \\
\hline Control group & 4 & $3(75 \%)$ & \\
\hline
\end{tabular}

While we only included patients who were willing to be tested for HIV, the cohort study by Ramadhan et al. [5] at our emergency department enrolled participants first and then asked if they were willing to be tested and receive their results. In that study, 250 (76.7\%) patients accepted testing for HIV, and among them, 98.8\% were ready to receive their test results when asked during counseling, demonstrating again the willingness of people who are asymptomatic to be tested.

The success of this intervention in Tanzania can be explained by the wide availability of mobile phones in our and other limited and middle-income countries which allows communication even in remote areas. The use of SMS (as opposed to email) meant that patients did not need to have internet access or incur any cost to receive the messages, eliminating the burden of buying internet data packages.

A total of $\$ 20,000$ was used in research tool development and \$170 used for domain name registration, sending messages, and hosting the research app online. The number needed to treat suggests this is a small cost compared to the cost incurred by the individual and government to treat advanced HIV/AIDS and its opportunistic infections. Used broadly, this type of technology would have a significant impact on the transmission and treatment of HIV.

The overall rate of follow-up for care in our cohort was $39.2 \%$, which is suboptimal. This shows the need for some type of intervention to improve compliance with care. Follow-up was significantly higher in the intervention group compared to the control group. Most of the patients were HIV-negative and were much more likely to follow-up for repeat screening if they received the SMS than if they were in the control group. HIVpositive patients had a good follow-up in both groups, but with only 9 people in total, the uncertainty surrounding the impact of the intervention is quite large. We would recommend that the Ministry of Health, Community Development, Gender, Elderly and Children plus the Government, and other stakeholders consider employing and funding SMS-based innovation to remind patients of important follow-up visits, not only in HIV but other chronic diseases, too.

\section{Study limitations and mitigation}

Our outcome of follow-up with recommended care was based on self-report. Self-reporting can be affected by social desirability bias. However, we would not expect the intervention group to be more likely to provide positive answers than the control group. Secondly, this was a single-center study; our results may not be generalizable to other hospitals in Tanzania. However, Muhimbili National Hospital is a tertiary-level referral hospital, receiving patients from all over the country. Finally, the small number of HIV-positive participants prohibited useful statistical testing on the effect of the intervention in this group.

In conclusion, text messaging is a feasible, efficient, and effective way to increase follow-up visits among individuals tested for HIV in LMICs. This study was able to show a significant difference between patients that receive a reminder text and those that do not. This relatively low-cost method can help in decreasing the burden of disease from HIV as well as other chronic illnesses in LMICs.

\section{Acknowledgements \\ The authors would like to acknowledge all the study participants and the Karuma family for their assistance in making this project successful.}

\section{Authors' contributions}

GMH conceived the idea and design of the study; acquired, analyzed, and interpreted the data; drafted the original manuscript; and revised the manuscript. HRS contributed to the conception and design of the study, data acquisition, entry, validation, and analysis and critically revised the manuscript. EW contributed to the design of the study, data validation, and analysis and critically revised the manuscript. SK contributed to the data validation, review, and analysis and revised the manuscript. JM contributed to the data review and analysis. RM contributed to the data validation, review, and analysis. SR contributed to the data review and analysis. WM contributed to the data validation, review, and analysis. FS contributed to the data analysis and review. MG contributed to the data validation and review. 
All authors contributed to the conception and design of the study, data validation, review, and analysis and also critically revised the manuscript. All authors read and approved the final manuscript.

\section{Funding}

The principal investigators used their own funds to support the data collection and logistics.

\section{Availability of data and materials}

The dataset supporting this study is available from the authors on request.

\section{Declarations}

Ethics approval and consent to participate

Permission to conduct the study was sought from relevant ethical committees at the Muhimbili University of Health and Allied Sciences (MUHAS) and the Muhimbili National Hospital (MNH) Research Committee. All patients were entered into the study after an informed consent agreement was signed. All patients received treatment as per standard hospital policies. The data obtained during the study was kept anonymous.

\section{Consent for publication}

Not applicable

\section{Competing interests}

The authors declare that they have no competing interests.

\section{Author details}

'Emergency Medicine Department, Muhimbili University of Health and Allied Sciences, P.O. Box 65001, Dar es Salaam, Tanzania. ${ }^{2}$ Emergency Medicine Department, Muhimbili National Hospital, Dar es Salaam, Tanzania. ${ }^{3}$ Department of Emergency Medicine, University of California San Francisco, San Francisco, CA, USA.

Received: 24 March 2021 Accepted: 12 November 2021

Published online: 14 December 2021

\section{References}

1. World Health Organization. WHO guideline. 2019 [cited 2020 Jul 24]. Available from: http://www.ncbi.nlm.nih.gov/books/NBK541902/

2. HIV/AIDS [Internet]. [cited 2020 Jun 8]. Available from: https://www.who.int/ news-room/fact-sheets/detail/hiv-aids

3. HIV and AIDS in Tanzania [Internet]. Avert. 2015 [cited 2020 Jun 8]. Available from: https://www.avert.org/professionals/hiv-around-world/sub-saharan-a frica/tanzania

4. Becker ML, Thompson LH, Pindera C, Bridger N, Lopez C, Keynan Y, et al. Feasibility and success of HIV point-of-care testing in an emergency department in an urban Canadian setting. Can J Infect Dis Med Microbiol. 2013;24(1):27-31. https://doi.org/10.1155/2013/164797.

5. Feasibility and yield of HIV screening among adult trauma patients presenting to an urban emergency department of a tertiary referral hospital in Tanzania | AIDS Research and Therapy | Full Text [Internet]. [cited 2020 Jun 8]. Available from: https://aidsrestherapy.biomedcentral.com/articles/1 0.1186/s12981-019-0223-5, 16, 1, 8

6. Tanzania, National AIDS Control Programme (Tanzania), editors. National comprehensive guidelines for HIV testing and counselling. Dar es Salaam: National Aids Control Programme (NACP); 2013. 73 p.

7. Bigna JJR, Noubiap JJN, Kouanfack C, Plottel CS, Koulla-Shiro S. Effect of mobile phone reminders on follow-up medical care of children exposed to or infected with HIV in Cameroon (MORE CARE): a multicentre, single-blind, factorial, randomised controlled trial. Lancet Infect Dis. 2014;14(7):600-8. https://doi.org/10.1016/S1473-3099(14)70741-8.

8. Mugo PM, Wahome EW, Gichuru EN, Mwashigadi GM, Thiong'o AN, Prins $H A B$, et al. Effect of text message, phone call, and in-person appointment reminders on uptake of repeat HIV testing among outpatients screened for acute HIV infection in Kenya: a randomized controlled trial. PloS One. 2016; 11(4):e0153612.

9. Zinski A, Westfall AO, Gardner LI, Giordano TP, Wilson TE, Drainoni M-L, et al. The contribution of missed clinic visits to disparities in HIV viral load outcomes. Am J Public Health. 2015;105(10):2068-75. https://doi.org/10.21 05/AJPH.2015.302695.
10. Norton BL, Person AK, Castillo C, Pastrana C, Subramanian M, Stout JE. Barriers to using text message appointment reminders in an HIV clinic. Telemed J E-Health Off J Am Telemed Assoc. 2014;20(1):86-9. https://doi. org/10.1089/tmi.2012.0275.

11. Paschen-Wolff MM, Restar A, Gandhi AD, Serafino S, Sandfort T. A systematic review of interventions that promote frequent HIV testing. AIDS Behav. 2019;23(4):860-74. https://doi.org/10.1007/s10461-019-02414-x.

12. Christopoulos KA, Riley ED, Tulsky J, Carrico AW, Moskowitz JT, Wilson L, et al. A text messaging intervention to improve retention in care and virologic suppression in a U.S. urban safety-net HIV clinic: study protocol for the Connect4Care (C4C) randomized controlled trial. BMC Infect Dis. 2014; 31(14):718.

13. Daher J, Vijh R, Linthwaite B, Dave S, Kim J, Dheda K, et al. Do digital innovations for HIV and sexually transmitted infections work? Results from a systematic review (1996-2017). BMJ Open. 2017;7(11):e017604. https://doi. org/10.1136/bmjopen-2017-017604.

14. Mayer JE, Fontelo P. Meta-analysis on the effect of text message reminders for HIV-related compliance. AIDS Care. 2017;29(4):409-17. https://doi.org/1 $0.1080 / 09540121.2016 .1214674$

15. Pop-Eleches C, Thirumurthy H, Habyarimana JP, Zivin JG, Goldstein MP, de Walque D, et al. Mobile phone technologies improve adherence to antiretroviral treatment in a resource-limited setting: a randomized controlled trial of text message reminders. AIDS Lond Engl. 2011;25(6):82534. https://doi.org/10.1097/QAD.0b013e32834380c1.

16. Bourne C, Knight V, Guy R, Wand H, Lu H, McNulty A. Short message service reminder intervention doubles sexually transmitted infection/HIV re-testing rates among men who have sex with men. Sex Transm Infect. 2011;87(3): 229-31. https://doi.org/10.1136/sti.2010.048397.

17. Menacho LA, Blas MM, Alva IE, Roberto OE. Short text messages to motivate HIV testing among men who have sex with men: a qualitative study in Lima. Peru. Open AIDS J. 2013;7(1):1-6. https://doi.org/10.2174/18746136013 07010001.

18. Sawe HR, Mfinanga JA, Mbaya KR, Kilindimo SS, Runyon MS. Trauma burden in Tanzania: a one-day survey of all district and regional public hospitals. 2017 [cited 2020 Jun 8]; Available from: http://dspace.muhas.ac.tz:8080/ xmlui/handle/123456789/2297

19. Research Randomizer [Internet]. [cited 2020 Jun 9]. Available from: https:// www.randomizer.org/

\section{Publisher's Note}

Springer Nature remains neutral with regard to jurisdictional claims in published maps and institutional affiliations.

Ready to submit your research? Choose BMC and benefit from

- fast, convenient online submission

- thorough peer review by experienced researchers in your field

- rapid publication on acceptance

- support for research data, including large and complex data types

- gold Open Access which fosters wider collaboration and increased citations

- maximum visibility for your research: over $100 \mathrm{M}$ website views per year

At $\mathrm{BMC}$, research is always in progress.

Learn more biomedcentral.com/submissions 